\title{
In this issue
}

Konstantza Tonova

Long-term preservation of $\alpha$-amylase

activity in highly concentrated

aqueous solutions of imidazolium

ionic liquid

DOI 10.1515/gps-2017-0016

Green Process Synth 2018; 7: 106-113

Batool Reyhaniyan Zavareh, Alireza

Salehirad and Saeed Mirdamadi

Effects of using the liquid phase method on the physicochemical, mechanical, and bioactivity properties of hydroxyapatite/calcium aluminate bioceramic nanocomposites

DOI 10.1515/gps-2016-0188

Green Process Synth 2018; 7: 122-131

Uyen Thi Phan Ngoc and Dai Hai

Nguyen

Synergistic antifungal effect of fungicide and chitosan-silver nanoparticles on Neoscytalidium dimidiatum

DOI 10.1515/gps-2016-0206

Green Process Synth 2018; 7: 132-138
Original article: The amylolytic activity is saved for a long time in highly concentrated aqueous solutions of imidazolium ionic liquid by adding an acetate salt.

Keywords: $\alpha$-amylase; acetate salt; enzyme activity; imidazolium bromide; ionic liquid.

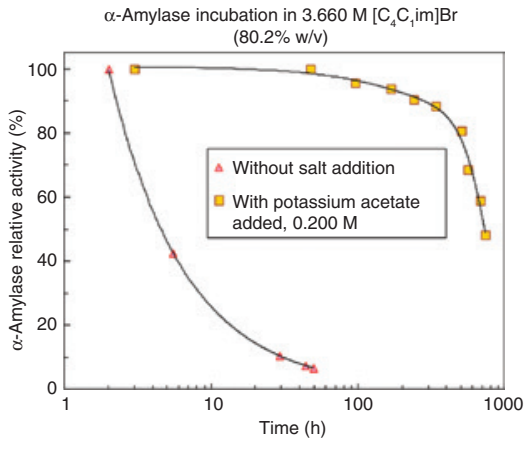

Original article: 80 wt.\% HA/20 wt.\% CA bioceramic nanocomposites were synthesized by using three different liquid phase green methods. According to the FE-SEM images of the nanocomposites, particle size distributions are in the range of $35 \mathrm{~nm}-110 \mathrm{~nm}$.

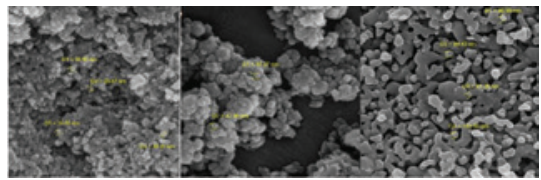

Keywords: bioceramic; calcium aluminate; hydroxyapatite; nanocomposite; synthesis method.

Original article: A novel drug delivery system of chitosan-silver nanoparticles with fungicide zineb was successfully developed against Neoscytalidium dimidiatum in dragon fruit.

Keywords: antifungal activity; chitosan; fungicide; Neoscytalidium dimidiatum; silver nanoparticle.

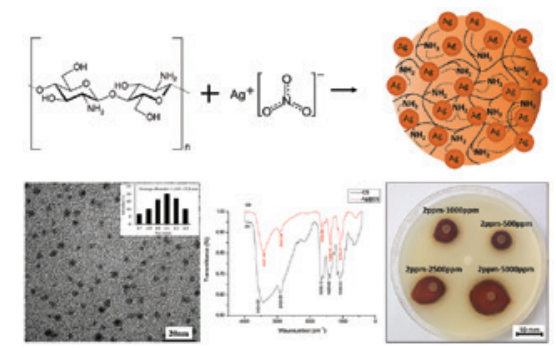


Hanh Ngoc Thi Le, Kiyoshi Imamura, Masakazu Furuta, Luu Van Boi and Yasuaki Maeda

Production of biodiesel from Vernicia montana Lour. oil using a co-solvent method and the subsequent evaluation of its stability during storage

DOI 10.1515/gps-2016-0215

Green Process Synth 2018; 7: 170-179
Original article: Biodiesel with high quality was produced using the co-solvent method with low energy consumption.

Keywords: biodiesel production; co-solvent method; iodine value; low energy consumption; Vernicia montana Lour. oil.

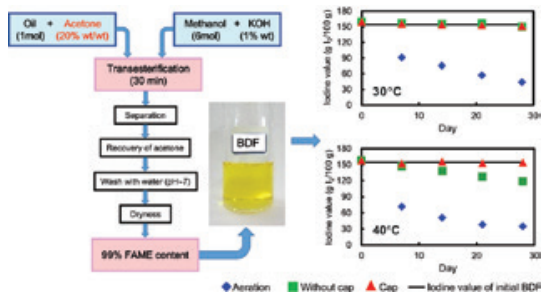

